\title{
Whole-exome sequencing identification of novel DNAH5 mutations in a young patient with primary ciliary dyskinesia
}

\author{
GEN KANO $^{1}$, HISASHI TSUJII ${ }^{1}$, KAZUHIKO TAKEUCHI $^{2}$, KANAME NAKATANI $^{3}$, \\ MAKOTO IKEJIRI $^{4}$, SATORU OGAWA ${ }^{5}$, HISAMI KUBO ${ }^{6}$, MIZUHO NAGAO ${ }^{7}$ and TAKAO FUJISAWA ${ }^{7}$ \\ ${ }^{1}$ Department of Pediatrics, Kyoto-Yamashiro General Medical Center, Kizugawashi, Kyoto 619-0214; \\ ${ }^{2}$ Department of Otorhinolaryngology, Head \& Neck Surgery; ${ }^{3}$ Division of Personalized Medicine; \\ ${ }^{4}$ Central Clinical Laboratories; ${ }^{5}$ Electron Microscopy Research Center, Mie University Graduate School of Medicine; \\ ${ }^{6}$ School of Medicine, Mie University, Tsu, Mie 514-8507; ${ }^{7}$ Department of Pediatrics, \\ Mie National Hospital, Tsu, Mie 514-0125, Japan
}

Received September 19, 2015; Accepted September 23, 2016

DOI: $10.3892 / \mathrm{mmr} .2016 .5871$

\begin{abstract}
Primary ciliary dyskinesia (PCD) is a rare genetic disorder caused by structural and/or functional impairment of cilia throughout the whole body. Early diagnosis of PCD is important for the prevention of long-term sequelae, however early diagnosis is a challenge due to the phenotypic heterogeneity of PCD. In the current study, the patient with PCD was diagnosed at nine years old following several efforts to control intractable airway symptoms. The patient experienced a chronic productive cough beginning in early childhood and had multiple episodes of pneumonia and otitis media with effusion and sinusitis. No situs inversus or other heterotaxias were reported. Serial chest X-rays exhibited persistent atelectasis and bronchiectasis in the right middle lobe. When the patient was nine years old, electron microscopy of his cilia and genetic analysis were conducted. Electron microscopy of a biopsy specimen from the nasal mucosa indicated loss of the outer dynein arms. Whole-exome analysis of the genome demonstrated the presence of compound heterozygous mutations in DNAH5: NM_001369.2:c.5983C >T, p.Arg1995X in exon 36 and NM_001369.2:c.9101delG, p.Gly3034ValfsX22 in exon 54; neither of which have been previously reported in the literature in a Japanese patient. Notably, this case is, to the best of our knowledge, the first reported case of PCD caused by the DNAH5 mutation in a Japanese patient.
\end{abstract}

Correspondence to: Professor Kazuhiko Takeuchi, Department of Otorhinolaryngology, Head \& Neck Surgery, Mie University Graduate School of Medicine, 2-174 Edobashi, Tsu, Mie 514-8507, Japan

E-mail: kazuhiko@clin.medic.mie-u.ac.jp

Key words: primary ciliary dyskinesia, atelectasis, DNAH5, whole exome sequencing

\section{Introduction}

Primary ciliary dyskinesia (PCD) is a rare genetic disorder of ciliary function and affects $\sim 1 / 20,000$ live births $(1 / 12,500-1 / 30,000)$ (1). Cilia serve critical roles throughout the human body and at all stages of life, including during left-right patterning of embryonic organs, the clearing of mucus and dirt from the respiratory tract, and the proper movement of sperm or ova; therefore, symptoms of PCD are diverse and include situs inversus, chronic oto-rhino-pulmonary infection and infertility. A large number $(>200)$ of genes code ciliary components, and the symptoms of PCD can vary between patients. This heterogeneity makes the diagnosis of PCD challenging, particularly when heterotaxia is absent and other symptoms are mild (2). This challenge is a problem, due to the fact that the later PCD is diagnosed, the worse the prognosis is (3). There is no gold-standard for diagnosis of PCD, and a comprehensive approach using both structural and functional analysis of the cilia and genetic analysis of causative genes is required. In the current study, a case of PCD in which the patient had been treated for 'intractable atelectasis with unknown origin' for nine years is reported. The present study highlighted the benefits of diagnostic strategies that include ultrastructural analysis accompanied by extended genetic analysis involving whole-exome sequencing.

\section{Patients and methods}

Chest computed tomography (CT) images were acquired by an Aquillion CT scanner (Toshiba Medical Systems Corporation, Otawara, Japan), with automatic exposure control (SD:12) at $120 \mathrm{kVp}$ with $5 \mathrm{~mm}$ section thicknesses. Nasal mucosa was obtained for electron microscopy. Under local anesthesia, a small amount of nasal mucosa was extracted from the patient's inferior turbinate. According to the methodology of Rubin (4), greater than 100 cilia from the patient were examined via electron microscopy (JEM-1011; JEOL, Tokyo, Japan). Nasal mucosa was also extracted from a subject with no nasal diseases and served as a normal control group. 


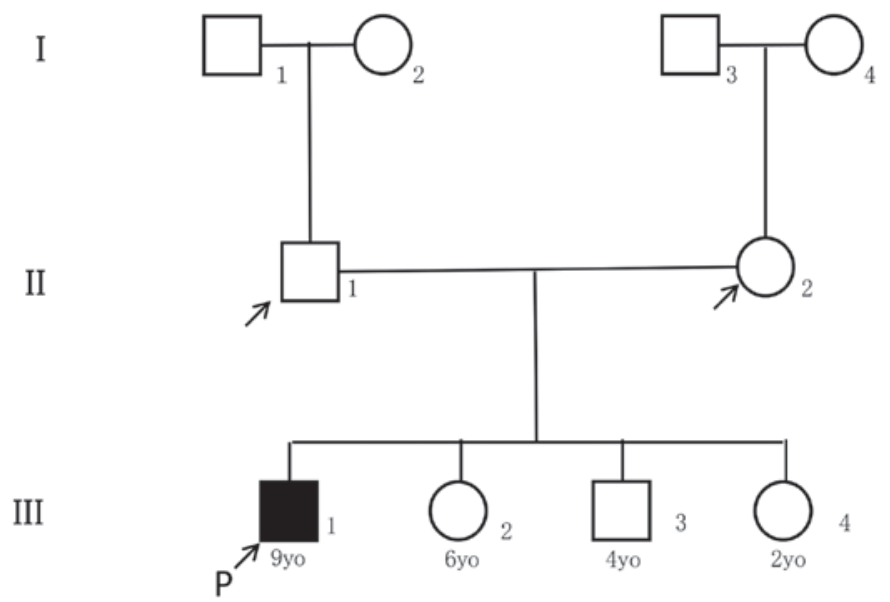

Figure 1. Family pedigree. The parents of the proband, his two younger sisters and his younger brother present with no respiratory illness.

Genetic analysis was approved by the Mie University University Ethics Committee (no. 1363), and written informed consent was obtained from the proband and each parent. Genomic DNA was extracted from peripheral blood samples taken from the forearm of each participant. Subsequently, known hot spots were sequenced in two candidate genes, DNAH5 and DNAII, due to the fact that a previous study observed mutations in DNAH5 or DNAII in approximately a third of all patients with PCD (5).

For the whole-exome sequencing, proband DNA was amplified with the Ion AmpliSeq ${ }^{\mathrm{TM}}$ Exome RDY Kit (Life Technologies; Thermo Fisher Scientific, Inc., Waltham, MA, USA), which targets more than $97 \%$ of human consensus coding sequences. After quality control thaws were performed with the Bioanalyzer High Sensitivity Chip (Agilent Technologies, Inc. Santa Clara, CA, USA) and emulsion polymerase chain reaction (PCR; Ion PI ${ }^{\mathrm{TM}} \mathrm{Hi}^{-\mathrm{Q}^{\mathrm{TM}}}$ OT2 200 kit; Life Technologies; Thermo Fisher Scientific, Inc.), samples were sequenced with a Proton PI chip version 3 and the Ion Proton semiconductor sequencer system (Life Technologies; Thermo Fisher Scientific, Inc.). Base calling, pre-processing of the reads, short read alignment and variant calling were performed with the Torrent Suite, the Torrent Variant Caller (version 4.6; Thermo Fisher Scientific, Inc.), and the default parameters recommended for the Ampliseq Exome panel (low stringency calling of germline variants, version, april 2014). Variant annotation was performed with Ion Reporter, version 4.6 (Life Technologies; Thermo Fisher Scientific, Inc.) and was data integrated from a variety of public databases.

Exome variant analysis was performed by filtering the whole variant list according to three criteria: i) Consistent autosomal recessive inheritance patterns, ii) novelty in comparison to human polymorphism databases [including the 1000 Genomes (http://www.1000genomes.org/) and dbSNP (http://www.ncbi. nlm.nih.gov/projects/SNP/)], and iii) functional significance. These analyses required the presence of at least one homozygous or two heterozygous changes occurring with an estimated frequency of $<0.01$.

Variants were validated via PCR and Sanger sequencing with the 3500 Series Genetic Analyzer (Thermo Fisher Scientific, Inc.). These tests were performed according to standard protocols specifically adapted to preclude technical artifacts and test for segregation. The primers used for the amplification were as follows: DNAH5 exon 36 F, 5'-CTTGTGTGCGTTTCA TGCCA-3'; DNAH5 exon 36 R, 5'-CTGCAACCGAGAGAA CTGGT-3'; DNAH5 exon 54 F, 5'-GATGATAACGGTGTT GGGGGAT-3', DNAH5 exon 54 R, 5'-GTAGCCCCGGAA AGGAGTAAAT-3'.

MutationTaster (http://mutationtaster.org/) and Polyphen-2 (http://genetics.bwh.harvard.edu/pph2/) analyses were conducted in order to predict the impact of variants in silico.

\section{Results}

Clinical information. The proband was a nine-year-old boy who was the offspring from non-consanguineous Japanese parents. His close relatives, specifically his parents, grandparents, two younger sisters and one younger brother, did not have a history of significant respiratory illness (Fig. 1). The first year of his life was uneventful, except that he experienced mild dyspnea and required low dose oxygen from the second to fourth days after birth. At one year of age, he was hospitalized for five days with a diagnosis of asthmatic bronchitis, with no significant lung infiltrations observed by X-ray (Fig. 2A). Since then, the patient experienced chronic nasal discharge and productive cough along with multiple episodes of sinusitis and/or otitis media. Atelectasis of the right lower lobe was first noted on a chest X-ray at three years of age, when he was admitted for acute pneumonia (Fig. 2B). Despite intense physiotherapy including positive expiratory pressure therapy or high frequency chest wall oscillation, the atelectasis remained unresolved (Fig. 2C and D). He was also diagnosed with asthma due to a frequent cough, leading to administration of inhaled corticosteroids and other asthma-specific therapies. At nine years of age, bronchiectasis was observed in the same lobe of the right lung by CT scanning (Fig. 3). Respiratory function tests at that point demonstrated mild airway obstruction with forced vital capacity of 1.641 (95.3\% predicted), forced expiratory volume $1.01 .18 \%$ (80.1\% predicted) and maximum midexpiratory flow $0.731 / \mathrm{sec}(37.2 \%$ predicted). All standard screening tests for immunodeficiency including serum immunoglobulin levels and T/B lymphocyte counts were normal; consequently, PCD was suspected, and the patient was referred to the Department of Otorhinolaryngology at our hospital. 
A

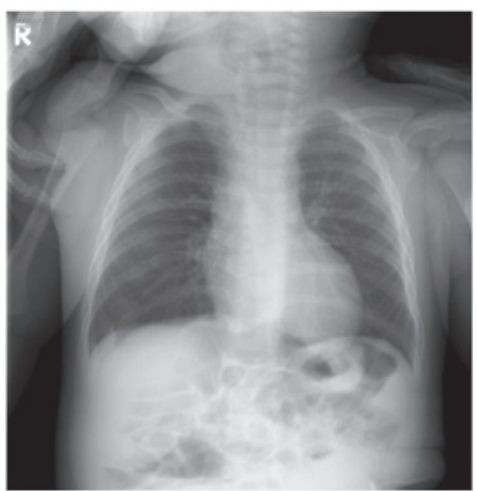

C

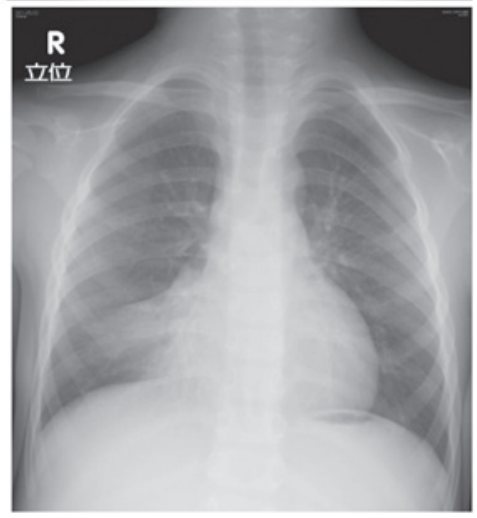

B

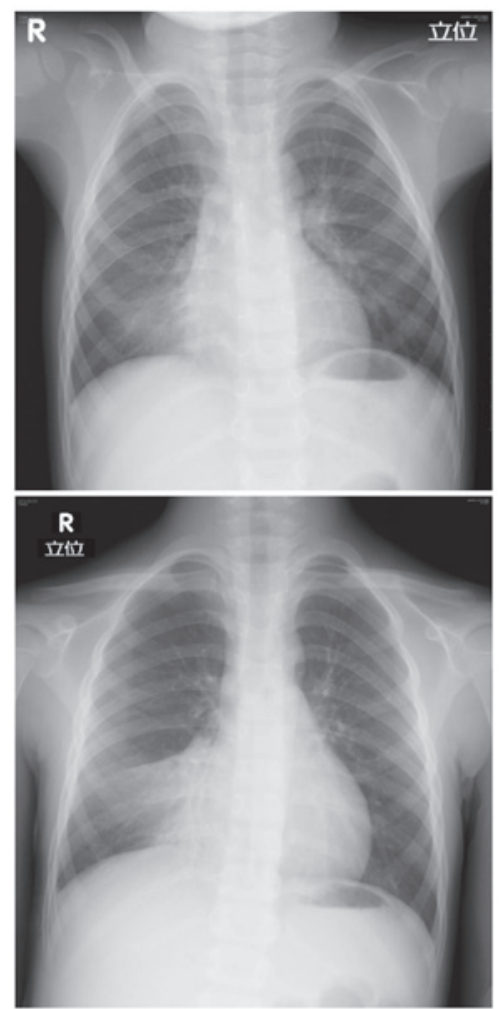

Figure 2. Serial chest X-rays of the patient. X-ray images at (A) one, (B) three, (C) seven and (D) nine years of age.

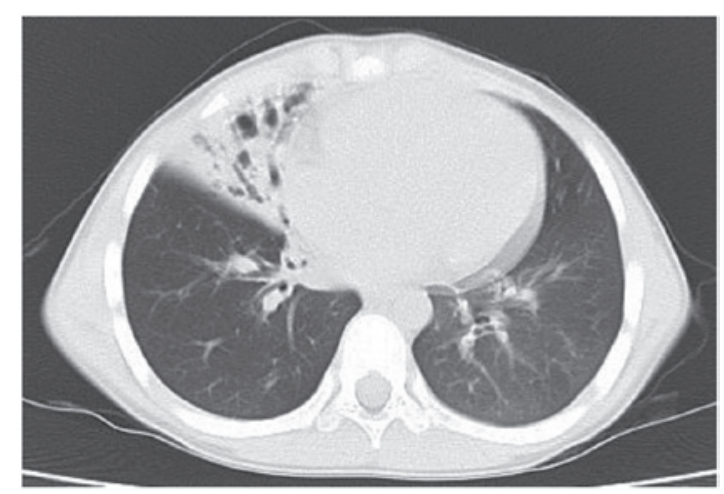

Figure 3. High resolution computed tomography scan of lung at nine years old shows atelectasis and bronchiectasis in the right middle lobe.

Otological examination revealed that the light reflex was missing from the right eardrum (Fig. 4A). Additionally, for the left eardrum, the posterosuperior quadrant was bulging, and the light reflex was missing (Fig. 4B). Pure-tone audiometry revealed bilateral conductive hearing loss with a hearing level of $35 \mathrm{~dB}$ in the right and $50 \mathrm{~dB}$ in the left (Fig. 4C). Tympanograms were type B (Fig. 4D) bilaterally; these observations were compatible with otitis media with effusion. Rhinological evaluation demonstrated that the nasal cavities were filled bilaterally with mucopurulent nasal secretions (Fig. 5A and B). Nasal X-ray revealed soft-tissue density bilaterally in the maxillary sinuses, suggesting chronic sinusitis and agenesis of the frontal sinuses (Fig. 5C and D). Nasal and exhaled nitric oxide (NO) was measured via an ANALYZER CLD $88^{\circledR}$ according to American Thoracic Society/European Respiratory Society recommendations (6); the fractional concentration of exhaled $\mathrm{NO}(\mathrm{FeNO})$ and nasal NO values were 10.0 and $0.2 \mathrm{ppb}$, respectively (data not shown). This low value of nasal NO was indicative of PCD (7).

Electron microscopy. Analysis of the specimen collected from the patient demonstrated shortened outer dynein arms (Fig. 6A-C); and this observation was compatible with PCD. In the normal control group (Fig. 6D), both the outer and inner dynein arms were observed.

Genetic analysis. No causative mutations were identified via conventional Sanger-based analyses of exons 34, 50, 63, 76 and 77 of DNAH5 (8) and exons 1, 13, 16, and 17 of DNAII (9). Based on these observations, whole-exome sequencing was conducted.

The whole-exome analysis of the proband genomic DNA identified two novel compound heterozygous mutations in DNAH5: NM_001369.2:c.5983C >T, p.Arg1995X in exon 36; 
A

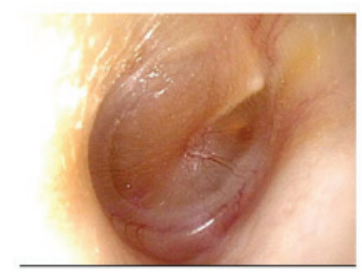

B

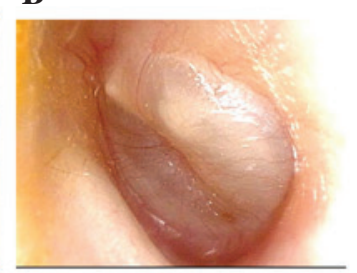

C

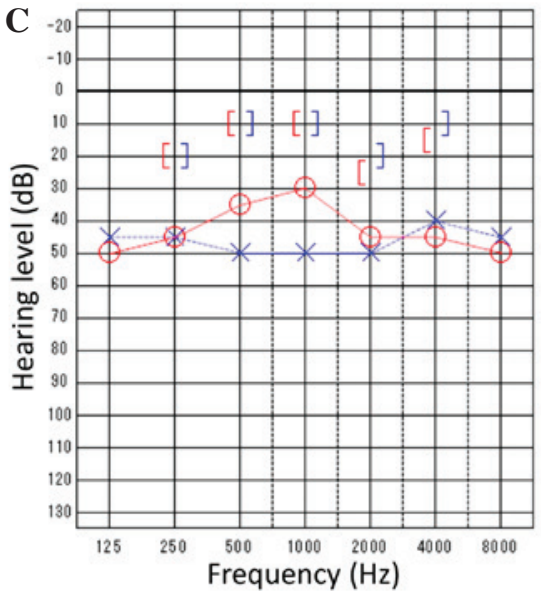

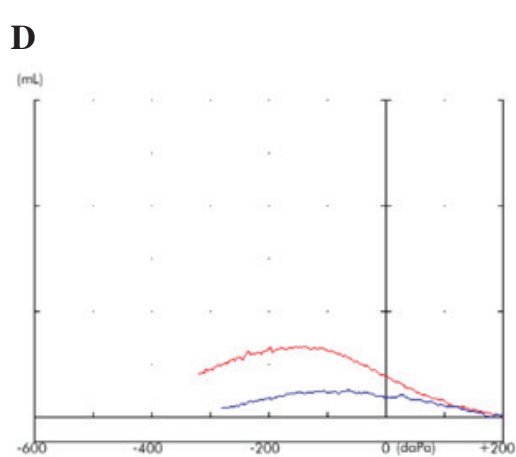

D

.400

Figure 4. Otological observations for the proband. Bilaterally, the eardrums were retracted (A, right; B, left). (C) The pure-tone audiogram indicated bilateral conductive hearing loss. (D) The tympanogram was type B bilaterally.

A

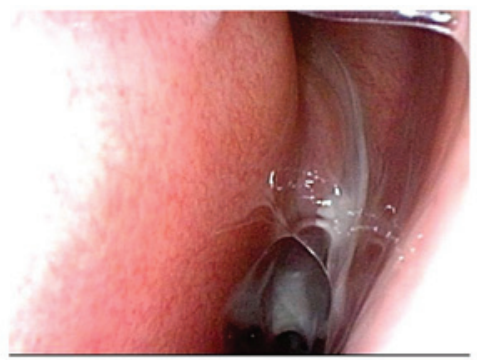

C

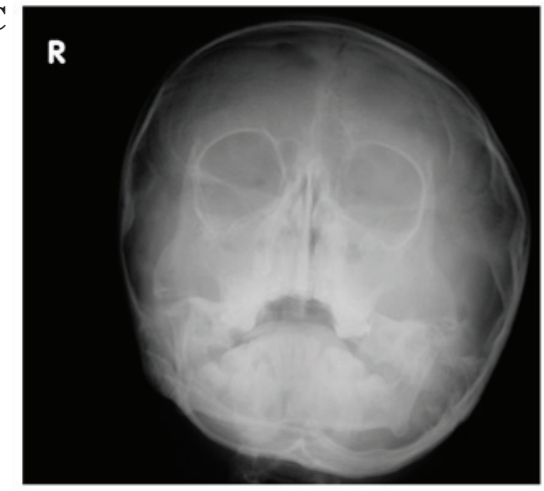

B

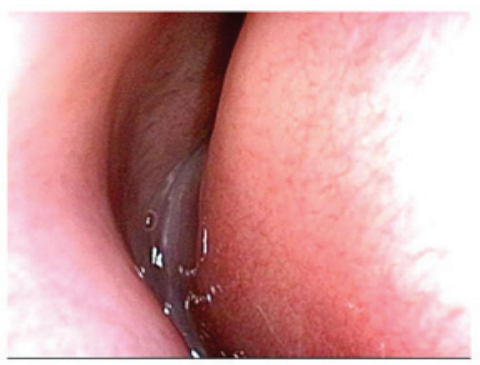

D

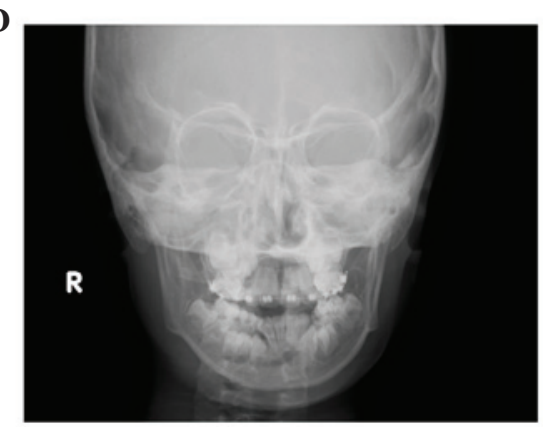

Figure 5. Rhinological observations for the proband. The nasal cavities were observed to be bilaterally filled with mucopurulent nasal secretions (A, right; B, left). Nose X-ray indicates (C) bilateral opacification of the maxillary sinuses and (D) clear agenesis of the frontal sinuses.

and NM_001369.2:c.9101delG, p.Gly3034ValfsX22 in exon 54. PolyPhen-2 analysis indicated that the p.Gly3034ValfsX22 mutation of DNAH5 was likely to be functionally damaging, with a score of 1.000. MutationTaster predicted that each of these DNAH5 mutations would cause nonsense-mediated mRNA decay.

Sanger sequencing confirmed the compound heterozygous mutations in DNAH5 identified by the whole-exome analysis in the proband (Fig. 7A); NM_001369.2:c.5983C >T, p.Arg1995X in exon 36 and NM_001369.2:c.9101delG, p.Gly3034ValfsX22 in exon 54. The patient's father carried only the former mutation (Fig. 7B), and his mother carried only the latter mutation (Fig. 7C). These observations confirmed that each mutation was inherited from a different parent.

\section{Discussion}

Pediatricians had followed this case for nine years before the diagnosis of PCD was made. Reportedly, the majority of patients with PCD are seen by physicians greater than 50 times prior to a diagnosis of PCD being made, and the mean age at PCD diagnosis is $10.9 \pm 14.4$ years (2). In addition, it takes markedly longer to make a diagnosis of PCD when situs inversus is absent. The value of this case is that sequential chest X-rays and CT scans were obtained over a long time period. An abnormality in a chest X-ray was observed at the age of three, however not at the age of one, and at a later date the patient had consolidation only in one lobe (the right middle lobe). For subjects 5-11 years old, Davis et al (10) observed that patients with inner dynein arm and central apparatus defects 
A

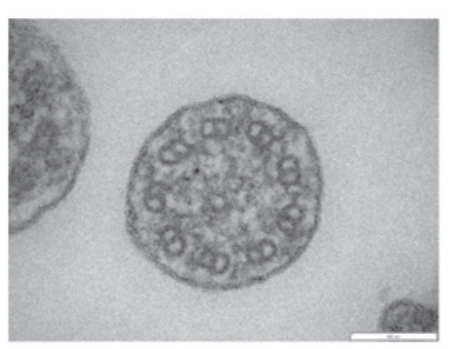

C

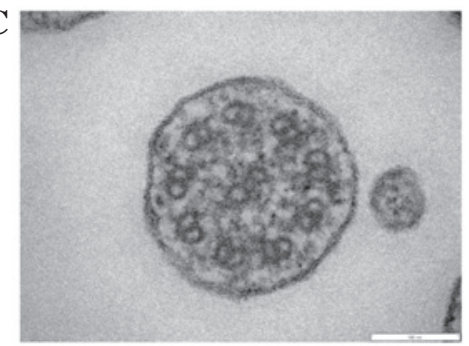

B

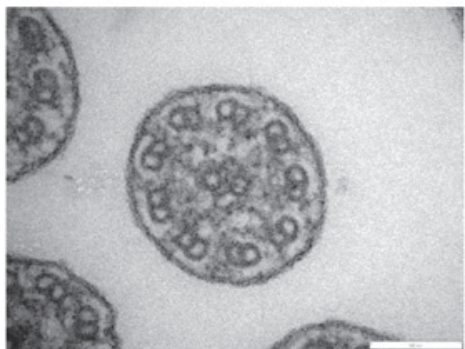

D

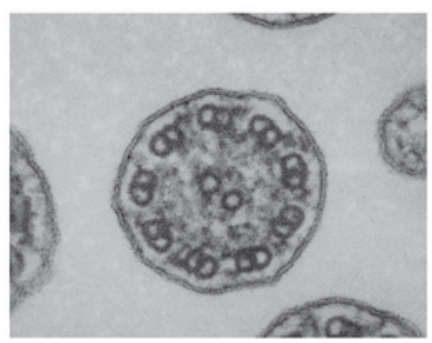

Figure 6. Electron microscopy for the proband. In the proband, (A) the central microtubules are unclear, (B) the outer dynein arms are shortened, (C) however the inner dynein arms are present. (D) In the control specimen, the inner and outer dynein arms were evident.

$\mathbf{A}$
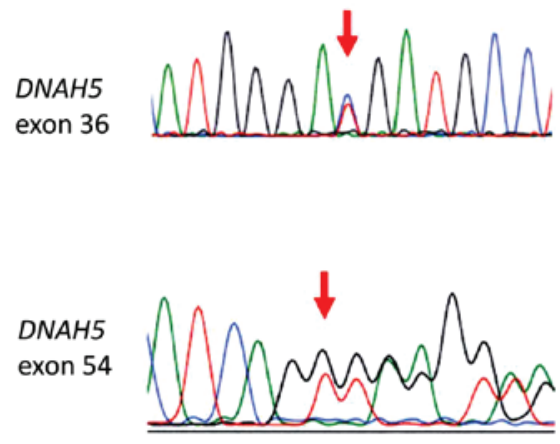

B

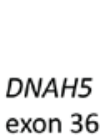

exon 36
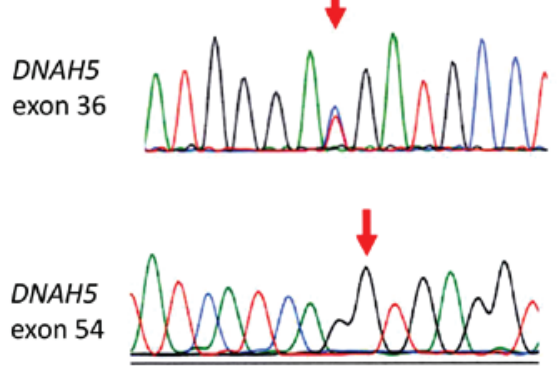

C
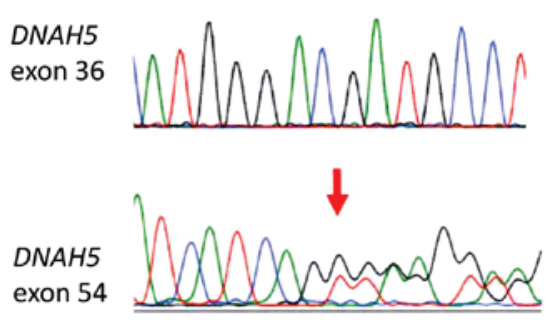

Figure 7. Whole-exome analysis of proband genomic DNA observed compound heterozygous mutations in DNAH5: NM_001369.2:c.5983C >T, p.Arg1995X in exon 36; and NM_001369.2:c.9101delG, p.Gly3034ValfsX22 in exon 54. (A) Sanger sequencing confirmed the two mutations in the proband. (B) The father of the proband carried only the former mutation, whereas the mother (C) carried only the latter. These finding were compatible with the normal autosomal inheritance pattern of compound heterozygous mutations.

with microtubular disorganization presented with more lobes with bronchiectasis (median, 5; $\mathrm{p}=0.0008$ ) and consolidation (median, 3; p=0.0001) than patients with outer dynein arm defects (median, 3 and 2, respectively). The proband in the current study had defects only in the outer dynein arms; therefore, he had a less severe lung pathology, which may partly explain the delay in a diagnosis of PCD.

According to Santamaria et al (11), the prevalence of lung changes on CT is as follows: Bronchiectasis, $80 \%$; peribronchial thickening, $80 \%$; mucous plugging, $75 \%$; parenchyma,
$65 \%$; and mosaic perfusion, $45 \%$. The lung CT of the patient in the present study indicated the presence of bronchiectasis and peribronchial thickening, the two most frequently occurring observations.

PCD is a Mendelian autosomal recessive and a genetically heterogeneous disorder. In a review article from 2013, Knowles et al (12) reported that PCD-causing mutations had been identified in 21 genes. The genes most commonly identified to result in PCD were DNAH5 (15-21\%), DNAII (2-9\%), DNAAF1 (LRRC50) (4-5\%), CCDC39 (2-10\%), 
CCDC4O (2-8\%), DNAH11 (6\%) and LRRC6 (3\%). Pathogenic mutations in 28 genes can reportedly lead to PCD, and these 28 genes reportedly account for approximately $70 \%$ of individuals affected with PCD (13). In the current study, electron microscopy identified shortened outer dynein arms. Regarding the association between ultrastructural phenotypes and genotypes, mutation of DNAH5, DNAI1, DNAI2, DNAL1, $C C D C 114, T X N D C 3$ or $A R M C 4$, which code for the structural components of the outer dynein arms, results in their loss.

In the present study, whole-exome analysis of proband genomic DNA identified compound heterozygous mutations in $D N A H 5$. At present, DNAH5 is reportedly the gene most often responsible for PCD; for example, mutations on both alleles of DNAH5 were identified in $15 \%$ of a clinically heterogeneous cohort of patients (14).

DNAH5 is a large gene comprising 79 exons and one alternative first exon and it encodes a heavy chain of the the outer dynein arm (8). A 1.5-kb partial cDNA representing DNAH5 was identified by Omran et al (15), and a full-length, $14 \mathrm{~kb}$ DNAH5 transcript was characterized by Olbrich et al (16).

Hornef et al (8) used haplotype analyses and/or sequencing to screen 109 caucasian PCD families originating from Europe and North America for the presence of DNAH5 mutations. They identified 33 novel and 2 known DNAH5 mutations. They observed clustering of mutations within five exons (exons 34, 50, 63, 76 and 77); and these five exons harbored $27(52 \%)$ of all 52 detected mutant alleles. Based on these observations, direct sequencing was conducted in the current study with these five exons to screen PCD-causing mutations. However, the two mutations in the examined patient were in exons 36 and 54: NM_001369.2:c.5983C >T, p.Arg1995X in exon 36 and NM_001369.2:c.9101delG, p.Gly3034ValfsX22 in exon 54.

Although DNAH5 mutations have been reported in PCD patients outside Japan, only one report of DNAH5 mutation in a Japanese patient was identified in PubMed. Tate et al (17) analyzed the case of a male neonate who exhibited three lobes of the left lung, asplenia and complex heart anomalies, who died $6 \mathrm{~h}$ subsequent to delivery. A heterozygous single nucleotide change (c.7829A $>$ G) was identified in exon 47 of $D N A H 5$, and this mutation resulted in the missense mutation of p.Glu2610Gly (17).

Zhang et al (18) performed exome capture and sequencing with samples from one affected individual and the unaffected parents from a Chinese Han community. They identified a homozygous mutation, c. 8030G $>$ A (Arg2677Gln), in DNAH5. This mutation was in exon 49, which is a known hot spot for PCD-causing mutations. Additionally, a patient from Germany had a missense mutation in $8029 \mathrm{C}>\mathrm{T}$, which resulted in Arg2677X (8).

To the best of our knowledge, both mutations identified in the current study are novel $(5,8,14,16)$. One is a nonsense mutation (NM_001369.2:c.5983C >T, p.Arg1995X) in exon 36; the other is a frame-shift mutation (NM_001369.2:c.9101delG, p.Gly3034ValfsX22) in exon 54. Of the PCD-causing mutations analyzed thus far, $85 \%$ are loss-of-function variants, and approximately $15 \%$ are conservative missense mutations (12). Among the 33 novel DNAH5 mutations detected by Hornef et al (8), 12 were nonsense mutations; 8 frame-shift mutations; 5 splicing variants and 8 missense mutations.
To predict the impact of these two newly identified variants in silico, MutationTaster (19) and Polyphen-2 (20) analyses were conducted. PolyPhen-2 analysis indicated that the p.Gly3034ValfsX22 mutation of DNAH5 was likely to be functionally damaging, with a score of 1.000 . MutationTaster assesses whether the predicted mutant proteins will be long- or short-lived and whether nonsense-mediated mRNA decay is likely to occur. MutationTaster predicted that each of these DNAH5 mutations would cause nonsense-mediated mRNA decay.

DNAH5 encodes ciliary dynein axonemal heavy chain 5 , a 4624-amino acid protein (16). The $\mathrm{N}$-terminal domain forms the stem domain of the outer dynein arm complex and is involved in interactions with other heavy, intermediate and light chains. The $\mathrm{C}$-terminal region that constitutes the globular head contains six conserved 6 p-loop domains and a conserved microtubule binding site (16). The first p-loop domain is known to bind and hydrolyze adenosine triphosphate (16). Both newly identified mutations were predicted to cause loss of function of the protein; therefore, it is likely that the two novel mutations are causal mutations resulting in PCD.

This is, to the best of our knowledge, the first report describing DNAH5 mutations in a Japanese patient with PCD. Whether there are relatively fewer patients with PCD among the Japanese compared with other ethnic groups is unclear, and requires investigation in future studies.

The present study reported a boy who had been followed by pediatricians for the first nine years of his life. Electron microscopy identified loss of the outer dynein arms in the cilia analyzed. Whole-exome analysis of the genomic DNA identified novel compound heterozygous mutations in DNAH5: NM_001369.2:c.5983C > T, p.Arg1995X in exon 36; and NM_001369.2:c.9101delG, p.Gly3034ValfsX22 in exon 54.

\section{Acknowledgements}

The present study was supported by Grant-in-Aid for General Scientific Research (C; grant nos. 25462662 and 16K11210) from the Ministry of Education, Sciences and Culture of Japan and the budget allocation from the director of Mie University Hospital $(2013,2014)$. The authors would like to thank Dr Issei Kobayashi and Dr Yuhko Kobayashi of Core-Lab, Graduate School of Regional Innovation Studies, Mie University (Mie, Japan) for their assistance with the genetic analysis. An English Language editing service provided by Forte Science Communications (Tokyo, Japan) was used (job no. R1506890).

\section{References}

1. Afzelius BA and Mossberg B: Immotile-cilia syndrome (primary ciliary dyskinesia), including Kartagener syndrome. In: The Metabolic and Molecular Bases of Inherited Disease. Scriver C, Beaudet A, Sly W and Valle D (eds). McGraw-Hill, New York, pp3943-3954, 1995.

2. Sommer JU, Schäfer K, Omran H, Olbrich H, Wallmeier J, Blum A, Hörmann K and Stuck BA: ENT manifestations in patients with primary ciliary dyskinesia: Prevalence and significance of otorhinolaryngologic co-morbidities. Eur Arch Otorhinolaryngol 268: 383-388, 2011.

3. Ellerman A and Bisgaard H: Longitudinal study of lung function in a cohort of primary ciliary dyskinesia. Eur Respir J 10: 2376-2379, 1997. 
4. Rubin BK: Immotile cilia syndrome (primary ciliary dyskinesia) and inflammatory lung disease. Clin Chest Med 9: 657-668, 1988.

5. Djakow J, Svobodová T, Hrach K, Uhlík J, Cinek O and Pohunek P: Effectiveness of sequencing selected exons of DNAH5 and DNAI1 in diagnosis of primary ciliary dyskinesia. Pediatr Pulmonol 47: 864-875, 2012.

6. American Thoracic Society; European Respiratory Society: ATS/ERS recommendations for standardized procedures for the online and offline measurement of exhaled lower respiratory nitric oxide and nasal nitric oxide, 2005. Am J Respir Crit Care Med 171: 912-930, 2005.

7. Narang I, Ersu R, Wilson NM and Bush A: Nitric oxide in chronic airway inflammation in children: Diagnostic use and pathophysiological significance. Thorax 57: 586-589, 2002

8. Hornef N, Olbrich H, Horvath J, Zariwala MA, Fliegauf M, Loges NT, Wildhaber J, Noone PG, Kennedy M, Antonarakis SE, et al: DNAH5 mutations are a common cause of primary ciliary dyskinesia with outer dynein arm defects Am J Respir Crit Care Med 174: 120-126, 2006

9. Zariwala MA, Leigh MW, Ceppa F, Kennedy MP, Noone PG Carson JL, Hazucha MJ, Lori A, Horvath J, Olbrich $\mathrm{H}$, et al: Mutations of DNAI1 in primary ciliary dyskinesia: Evidence of founder effect in a common mutation. Am J Respir Crit Care Med 174: 858-866, 2006

10. Davis SD, Ferkol TW, Rosenfeld M, Lee HS, Dell SD, Sagel SD, Milla C, Zariwala MA, Pittman JE, Shapiro AJ, et al: Clinical features of childhood primary ciliary dyskinesia by genotype and ultrastructural phenotype. Am J Respir Crit Care Med 191: 316-324, 2015

11. Santamaria F, Montella S, Tiddens HA, Guidi G, Casotti V, Maglione M and de Jong PA: Structural and functional lung disease in primary ciliary dyskinesia. Chest 134: 351-357, 2008.

12. Knowles MR, Daniels LA, Davis SD, Zariwala MA and Leigh MW: Primary ciliary dyskinesia. Recent advances in diagnostics, genetics, and characterization of clinical disease. Am J Respir Crit Care Med 188: 913-922, 2013.
13. Knowles MR, Ostrowski LE, Leigh MW, Sears PR, Davis SD, Wolf WE, Hazucha MJ, Carson JL, Olivier KN, Sagel SD, et al: Mutations in RSPH1 cause primary ciliary dyskinesia with a unique clinical and ciliary phenotype. Am J Respir Crit Care Med 189: 707-717, 2014.

14. Failly M, Bartoloni L, Letourneau A, Munoz A, Falconnet E, Rossier C, de Santi MM, Santamaria F, Sacco O, DeLozier-Blanchet CD, et al: Mutations in DNAH5 account for only $15 \%$ of a non-preselected cohort of patients with primary ciliary dyskinesia. J Med Genet 46: 281-286, 2009.

15. Omran H, Häffner K, Völkel A, Kuehr J, Ketelsen UP, Ross UH, Konietzko N, Wienker T, Brandis M and Hildebrandt F: Homozygosity mapping of a gene locus for primary ciliary dyskinesia on chromosome 5p and identification of the heavy dynein chain DNAH5 as a candidate gene. Am J Respir Cell Mol Biol 23: 696-702, 2000.

16. Olbrich H, Häffner K, Kispert A, Völkel A, Volz A, Sasmaz G, Reinhardt R, Hennig S, Lehrach H, Konietzko N, et al: Mutations in DNAH5 cause primary ciliary dyskinesia and randomization of left-right asymmetry. Nat Genet 30: 143-144, 2002.

17. Tate G, Tajiri T, Kishimoto K and Mitsuya T: A novel mutation of the axonemal dynein heavy chain gene 5 (DNAH5) in a Japanese neonate with asplenia syndrome. Med Mol Morphol 48: 116-122, 2015.

18. Zhang J, Guan L, Wen W, Lu Y, Zhu Q, Yuan H, Chen Y, Wang $\mathrm{H}$, Zhang $\mathrm{J}$ and $\mathrm{Li} \mathrm{H}$ : A novel mutation of DNAH5 in chronic rhinosinusitis and primary ciliary dyskinesia in a Chinese family. Eur Arch Otorhinolaryngol 271: 1589-1594, 2014.

19. Schwarz JM, Cooper DN, Schuelke M and Seelow D: MutationTaster2: Mutation prediction for the deep-sequencing age. Nat Methods 11: 361-362, 2014.

20. Adzhubei I, Schmidt S, Peshkin L, Ramensky VE, Gerasimova A, Bork P, Kondrashov AS and Sunyaev SR: A method and server for predicting damaging missense mutations. Nat Methods 7: 248-249, 2010. 